\title{
A SYMMETRIC HOMOTOPY AND HYBRID POLYNOMIAL SYSTEM SOLVING METHOD FOR MIXED TRIGONOMETRIC POLYNOMIAL SYSTEMS
}

\author{
BO DONG, BO YU, AND YAN YU
}

\begin{abstract}
A mixed trigonometric polynomial system, which rather frequently occurs in applications, is a polynomial system where every monomial is a mixture of some variables and sine and cosine functions applied to the other variables. Polynomial systems transformed from the mixed trigonometric polynomial systems have a special structure. Based on this structure, a hybrid polynomial system solving method, which is more efficient than random product homotopy method and polyhedral homotopy method in solving this class of systems, has been presented. Furthermore, the transformed polynomial system has an inherent partially symmetric structure, which cannot be adequately exploited to reduce the computation by the existing methods for solving polynomial systems. In this paper, a symmetric homotopy is constructed and, combining homotopy methods, decomposition, and elimination techniques, an efficient symbolic-numerical method for solving this class of polynomial systems is presented. Preservation of the symmetric structure assures us that only part of the homotopy paths have to be traced, and more important, the computation work can be reduced due to the existence of the inconsistent subsystems, which need not to be solved at all. Exploiting the new hybrid method, some problems from the literature and a challenging practical problem, which cannot be solved by the existing methods, are resolved. Numerical results show that our method has an advantage over the polyhedral homotopy method, hybrid method and regeneration method, which are considered as the state-of-art numerical methods for solving highly deficient polynomial systems of high dimension.
\end{abstract}

\section{INTRODUCTION}

A mixed trigonometric polynomial system (abbreviated by MTPS) is a polynomial system whose monomial is mixed by some variables and sine and cosine functions applied to the other variables. This class of systems occurs in many fields of science and engineering, such as neurophysiology [23, kinematics [4, 23], PUMA

Received by the editor October 21, 2011 and, in revised form, July 3, 2012.

2010 Mathematics Subject Classification. Primary 13P15, 65H20; Secondary 65H10, 14Q99, 68W30.

Key words and phrases. Mixed trigonometric polynomial system, polynomial system, symmetry, homotopy method, hybrid algorithm, symbolic-numeric computation.

The first author was supported in part by the National Natural Science Foundation of China (Grant No. 11101067), TianYuan Special Funds of the National Natural Science Foundation of China (Grant No. 11026164) and the Fundamental Research Funds for the Central Universities.

The second author's research was supported by Major Research Plan of the National Natural Science Foundation of China (Grant No. 91230103) and the National Natural Science Foundation of China (Grant No. 11171051). 
robot [18, 19], etc. Formally, An MTPS can be shown as follows:

$$
F(y, \theta)=\left(f_{1}(y, \theta), \ldots, f_{n+m}(y, \theta)\right)^{T}=0,
$$

where $y=\left(y_{1}, \ldots, y_{n}\right), \theta=\left(\theta_{1}, \ldots, \theta_{m}\right)$, and for $1 \leq i \leq n+m$,

$$
f_{i}(y, \theta)=\sum_{\alpha \in S_{i}} b_{i \alpha} y^{\beta} \sin ^{\mu} \theta \cos ^{\nu} \theta+c_{i}^{1},
$$

where $\alpha=(\beta, \mu, \nu), S_{i} \subset \mathcal{N}^{n+2 m} \backslash\{0\}$ and

$$
\left\{\begin{aligned}
y^{\beta} & =y_{1}^{\beta_{1}} \cdots y_{n}^{\beta_{n}}, \\
\sin ^{\mu} \theta & =\sin ^{\mu_{1}} \theta_{1} \cdots \sin ^{\mu_{m}} \theta_{m}, \\
\cos ^{\nu} \theta & =\cos ^{\nu_{1}} \theta_{1} \cdots \cos ^{\nu_{m}} \theta_{m} .
\end{aligned}\right.
$$

Example 1.1. This is a challenging practical problem we meet in signal processing of sonar and radar, which is also the original motivation of this research:

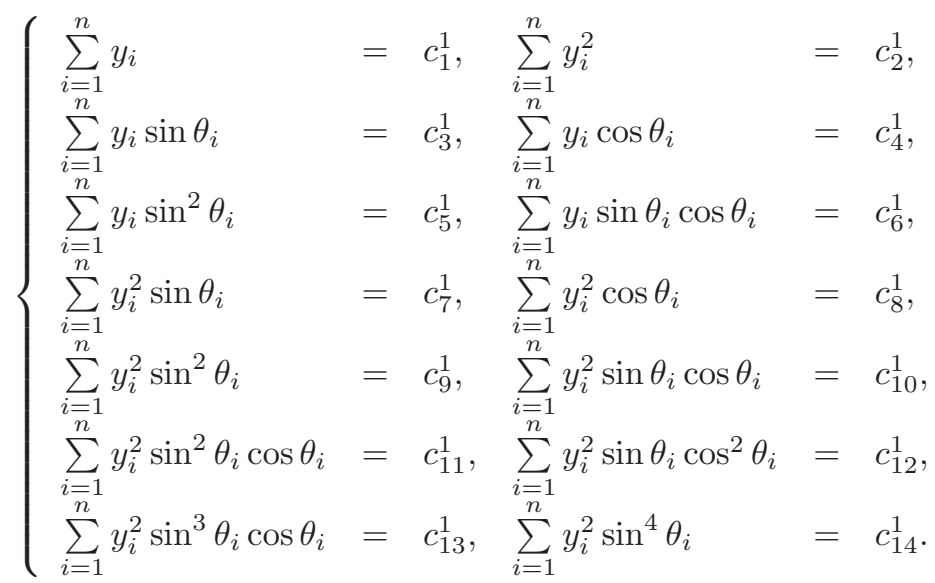

where, $n=3$ to 7 (if $n<7$, then only the first $2 n$ equations are needed).

When $m=0$, the MTPSs in (1.1), (1.2) and (1.3) is a polynomial system, so polynomial systems can be regarded as a special class of MTPS's, on the other hand, substituting occurrences of $y_{i}, \sin \theta_{j}$ and $\cos \theta_{j}$ with new variables $x_{i}, x_{n+j}$ and $x_{n+m+j}$ and adding quadratic equations of the form $x_{n+j}^{2}+x_{n+m+j}^{2}-1=0$, where $i=1, \ldots, n, j=1, \ldots, m$, the MTPS $F(y, \theta)=0$ in (1.1), (1.2) and (1.3) can be transformed to a polynomial system with special structures as follows:

$$
P(x)=\left(\begin{array}{c}
p_{1}(x)+c_{1}^{1} \\
\cdots \\
p_{n+m}(x)+c_{n+m}^{1} \\
x_{n+1}^{2}+x_{n+m+1}^{2}-1 \\
\cdots \\
x_{n+m}^{2}+x_{n+2 m}^{2}-1
\end{array}\right)=0,
$$

where

$$
p_{i}(x)=\sum_{\alpha \in S_{i}} b_{i \alpha} x^{\alpha} .
$$


Example 1.2 (Example 1.1 continued). This is the polynomial system transformed from the MTPS in Example 1.1.

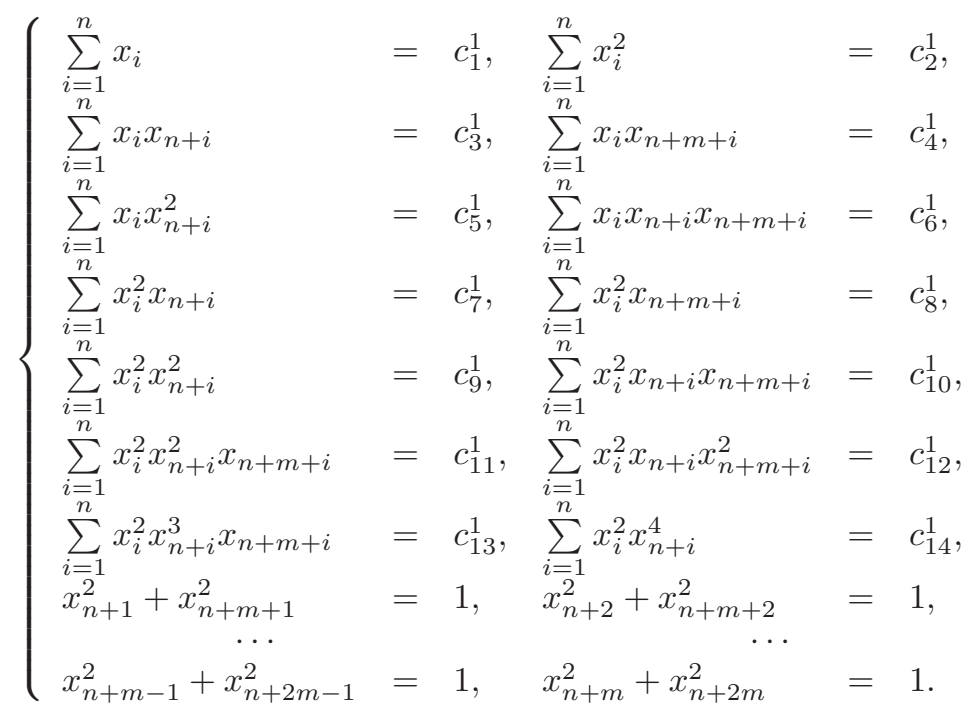

1.1. Homotopy methods for solving polynomial systems. Homotopy method is an efficient numerical method for solving polynomial systems. The homotopy method is used to define a polynomial system $Q(x)=0$, known as the start system, and then follows the curves parameterized by the variable $t$ consisting of the solutions of

$$
H(x, t)=(1-t) Q(x)+t P(x)
$$

to get the solutions of the target system $P(x)=0$. If the start system $Q(x)=0$ is chosen correctly, the following three properties hold [13:

Triviality: The solutions of $H(x, 0)=Q(x)=0$ are known.

Smoothness: The solution set of $H(x, t)=0$ for all $0 \leq t<1$ consists of a finite number of smooth paths, each parameterized by $t \in[0,1)$.

Accessibility: Every isolated solution of $H(x, 1)=0$ can be reached by some paths originating at a solution of $H(x, 0)=0$.

When these three properties hold, tracing numerically homotopy paths in the variable $t$ from 0 to 1 , we can find all isolated solutions of $P(x)=0$.

Standard homotopies, which generate Bézout number of homotopy paths, are given in [12,20,30,33. Theoretically, standard homotopy can be applied to find all isolated solutions of any polynomial systems. However, polynomial systems arising in practice are often deficient, that is, its actual number of solutions is less than the Bézout number. For deficient systems, standard homotopies generate many extraneous paths that tend to infinity and hence make wasted computation. Therefore, studies on homotopy methods for solving polynomial systems are now focused on exploiting sparsity of the system, that is, constructing better homotopy such that the number of homotopy paths to be traced is proportional to the actual number of isolated solutions of the target system. Many homotopies for deficient polynomial systems have been constructed, the list is hardly exhaustive; see discussions in [5, 8, 11, 14, 16, 19, 21, 22, 25, 28, 29, 32, and references contained therein. 
For its essential complexity, the polynomial system solving problem is far from satisfactorily resolved. The above mentioned homotopy methods still cannot be applied to efficiently solve practical polynomial systems of moderate size. As an example, for polynomial systems in (1.7), when $n=5,6,7$, calculated by the software PHCpack 24, Bézout numbers are 331776, 16588800, 1194393600 and the minimal multi-homogeneous Bézout numbers are 73728, 3736576, 270811136, respectively. It is unsuitable to solve this class of polynomial systems by the standard homotopy methods, multi-homogeneous homotopy methods or other random product homotopy methods, and the polyhedral homotopy methods are also difficult to apply because computation of the mixed subdivision takes too much time. Further effort needs to be made to solve polynomial systems more efficiently by exploiting their special structure.

1.2. Regeneration methods for solving polynomial systems. The method in [6] uses the regeneration technique to solve the polynomial systems. It is a multistage method, and in each stage, the equations are introduced one-by-one or in groups, the solutions of the current stage can be extended to the next stage until all the solutions of the entire polynomial system are obtained.

Precisely speaking, to find all isolated solutions of the target polynomial system $P(x)=\left(p_{1}(x), \ldots, p_{n}(x)\right)^{\top}=0$, one should first reorder all polynomials according to some rules, and then, for every polynomial $p_{i}(x)$, specify a linear product decomposition $q_{i}(x)=q_{i 1}(x) \cdots q_{i d_{i}}(x)$ and a generic product member $r_{i}(x)$, which is a random linear combination of all variables in polynomial $p_{i}(x)$ and 1 . Without loss of generality, suppose the reordered system is still $P(x)=\left(p_{1}(x), \ldots, p_{n}(x)\right)^{\top}=0$ and all polynomials are partitioned into $k$ groups, and the index is $0=m_{0} \leq m_{1} \leq$ $\ldots \leq m_{k}=n$ :

- Step 1. Use the numerical linear algebra to solve the polynomial system

$$
\left(q_{1}(x), \ldots, q_{m_{1}}(x), r_{m_{1}+1}(x), \ldots, r_{n}(x)\right)^{\top},
$$

then take this system as the start system to get all solutions of

$$
\left(p_{1}(x), \ldots, p_{m_{1}}(x), r_{m_{1}+1}(x), \ldots, r_{n}(x)\right)^{\top} .
$$

- Step 2. For $i=2, \ldots, n$, do the following:

Taking

$$
\left(p_{1}(x), \ldots, p_{m_{i-1}}(x), r_{m_{i-1}+1}(x), \ldots, r_{m_{i}}(x), r_{m_{i}+1}(x), \ldots, r_{n}(x)\right)^{\top} .
$$

as the start system to get all isolated solutions of

$$
\left(p_{1}(x), \ldots, p_{m_{i-1}}(x), q_{m_{i-1}+1}(x), \ldots, q_{m_{i}}(x), r_{m_{i}+1}(x), \ldots, r_{n}(x)\right)^{\top},
$$

then taking this system as the start system to get all isolated solutions of

$$
\left(p_{1}(x), \ldots, p_{m_{i-1}}(x), p_{m_{i-1}+1}(x), \ldots, p_{m_{i}}(x), r_{m_{i}+1}(x), \ldots, r_{n}(x)\right)^{\top} .
$$

The regeneration method is a general method used to solve deficient polynomial systems, and for a different class of polynomial systems, the rules on the manner of reordering equations and introducing equations in each step may be totally different. To solve polynomial systems transformed from MTPS's more efficiently, the rules in [6] are unsuitable, and should be considered more specifically. On the other hand, in [6], the authors did not discuss how to solve the polynomial systems more efficiently by exploiting their (partially) symmetric structure. However, the applications of 
the (partial) symmetry to reduce the computational work is our main goal in this paper.

1.3. Hybrid polynomial system solving methods for MTPS's. Polynomial systems transformed from MTPS's have a special structure: the last $m$ equations are of the form

$$
x_{n+i}^{2}+x_{n+m+i}^{2}-1=0, i=1, \ldots, m .
$$

In [31], by exploiting this special structure, we proposed a hybrid polynomial system solving method for MTPS. It hybridizes the homotopy method and some symbolic computation techniques, such as variable substitution, variable elimination, and some reduction techniques. The homotopy we constructed is also hybrid, it combines linear product homotopy and coefficient parameter homotopy [21] or cheater's homotopy 9]. In our experience, for the polynomial systems transformed from MTPS's, the hybrid method is more efficient than the multi-homogeneous homotopy method or the polyhedral homotopy method, which is the state-of-art numerical method for solving deficient polynomial systems.

Besides the above special structure, polynomial systems transformed from MTPS's have a special structure. Its lower part consists of $m$ equations of the form

$$
x_{n+i}^{2}+x_{n+m+i}^{2}-1=0(1 \leq i \leq m)
$$

and possesses an inherent symmetry of variable exchanges,

$$
\begin{aligned}
\left\{x_{n+i}\right\} & \leftrightarrow\left\{x_{n+m+i}\right\}, \\
\left\{x_{n+i}, x_{n+m+i}\right\} & \leftrightarrow\left\{x_{n+j}, x_{n+m+j}\right\}, \text { or } \\
\left\{x_{n+i}, x_{n+m+i}\right\} & \leftrightarrow\left\{x_{n+m+j}, x_{n+j}\right\},
\end{aligned}
$$

where $S_{i} \leftrightarrow S_{j}$ denotes exchanging places of all variables in $S_{i}$ and the corresponding variables in $S_{j}$ do not change these $m$ equations except for their order. For a symmetric polynomial system, its solutions can be divided to some equivalent classes, and all solutions in a class can be generated in some rules by any solution, called representative solution, in it. If the constructed homotopy can keep the symmetry of the target polynomial system, then only the representative solution path in each equivalent class should be traced, and hence the computational work can be decreased considerably.

1.4. Homotopy methods for symmetric polynomial systems. In many cases, polynomial systems arising in practical problems are symmetric, and some symmetric homotopy methods have been published.

In [17, Meravý provided a sign-symmetric homotopy for solving polynomial systems which have symmetric solution sets and gave conditions on start systems in the general cases. However, this homotopy was constructed according to the classic theorem of Bézout which is not suitable for the deficient polynomial systems. In [15], Li and Sauer applied the random product homotopy to what they called a self-symmetric polynomial system, which belongs to polynomial systems that are suitable to apply the random product homotopy to solve. In [26], for the polynomial systems with a $(G, V, W)$-symmetric structure, Verschelde and Cools gave an algorithm to verify whether a given system is $(G, V, W)$-symmetric and presented how to construct a $(G, V, W)$-symmetric homotopy. Applying this homotopy, only the representative solution paths have to be traced. In [27, symmetric polyhedral homotopies were constructed to deal with $(G, V, W)$-symmetric polynomial systems. 
The existing methods for symmetric polynomial systems are mainly focused on the specially symmetric structure, for the more generally symmetric structure, these methods are hard to be applied or generalized. The symmetric homotopy methods mentioned above cannot adequately take advantage of the specially symmetric structure mentioned in Section 1.3 .

In this paper, exploiting the special structure and the symmetry in (1.8), a homotopy, which is a combination of a special coefficient-parameter homotopy and the random product homotopy and keeps the symmetry of its lower part, is constructed. Combining the homotopy method, decomposition and elimination techniques, an efficient symbolic-numerical method, called the symmetric hybrid method, for solving this class of polynomial systems are presented.

The paper is organized as follows. The symmetric hybrid method for solving polynomial systems coming from MTPS's is given in Section 2, and also a theorem to ensure applying our symmetric homotopy can find all isolated solutions of the target system are presented. The precise solution procedure and the advantages of our symmetric hybrid method are described in Section 3 The comparison of our symmetric hybrid method with other methods for solving MTPS's are discussed in Section 4. Numerical examples and applications are given in Section 5]

\section{Symmetric HYBRid METHOD FOR POLYNOMial SYSTEMS TRANSFORMED FROM MTPS'S}

Motivated by 31] and the existing symmetric homotopy methods, we present a symmetric homotopy and hybrid method to solve the transformed polynomial systems more efficiently.

2.1. Symmetric hybrid homotopy. To keep the symmetric structure of polynomial systems in (1.5), we construct the following symmetric homotopy:

$$
H(x, t)=\gamma(1-t) Q(x)+t P(x),
$$

where

$$
(2.2) Q(x)=\left(\begin{array}{c}
p_{1}(x)+c_{1}^{0} \\
\cdots \\
p_{n+m}(x)+c_{n+m}^{0} \\
\left(s_{1} x_{n+1}+s_{2} x_{n+m+1}+s_{0}\right)\left(s_{2} x_{n+1}+s_{1} x_{n+m+1}+s_{0}\right) \\
\cdots \\
\left(s_{1} x_{n+m}+s_{2} x_{n+2 m}+s_{0}\right)\left(s_{2} x_{n+m}+s_{1} x_{n+2 m}+s_{0}\right)
\end{array}\right),
$$

and $\gamma, c_{1}^{0}, \ldots, c_{n+m}^{0}, s_{0}, s_{1}, s_{2}$ are all random nonzero complex numbers.

The start system $Q(x)=0$ can be decomposed into $2^{m}$ subsystems,

$$
Q_{\sigma}(x)=\left(\begin{array}{c}
p_{1}(x)+c_{1}^{0} \\
\cdots \\
p_{n+m}(x)+c_{n+m}^{0} \\
s_{\sigma_{1}} x_{n+1}+s_{3-\sigma_{1}} x_{n+m+1}+s_{0} \\
\cdots \\
s_{\sigma_{m}} x_{n+m}+s_{3-\sigma_{m}} x_{n+2 m}+s_{0}
\end{array}\right)=0
$$

where $\sigma \in \Sigma:=\left\{\left(\sigma_{1}, \ldots, \sigma_{m}\right) \mid \sigma_{i} \in\{1,2\}\right\}$.

Remark 2.1. The last $m$ equations of the start system (2.2) have the same symmetric structure as those of the target system. If the target system is symmetric, then 
in the process of tracing homotopy paths, only the representative solution paths have to be traced, and thus the computational work can be reduced.

The following example is to show the importance of applying the symmetric structure of the target system, and furthermore, it shows our symmetric homotopy has more extensive applications in exploiting the symmetry of the target system:

Example 2.1. Consider the following polynomial system

$$
P(x)=\left(\begin{array}{c}
x_{1}+x_{2}+x_{3}+x_{4}-1 \\
x_{1} x_{2}+x_{3} x_{4}-2 \\
x_{1}^{2}+x_{2}^{2}-1 \\
x_{3}^{2}+x_{4}^{2}-1
\end{array}\right)=0 .
$$

This system has eight nonsingular isolated solutions, and it is not difficult to observe the following property: if $\left(a_{1}, a_{2}, a_{3}, a_{4}\right)$ is a solution of $P(x)=0$, then

$$
\begin{gathered}
\left(a_{1}, a_{2}, a_{4}, a_{3}\right),\left(a_{2}, a_{1}, a_{3}, a_{4}\right),\left(a_{2}, a_{1}, a_{4}, a_{3}\right) \\
\left(a_{3}, a_{4}, a_{2}, a_{1}\right),\left(a_{3}, a_{4}, a_{1}, a_{2}\right),\left(a_{4}, a_{3}, a_{2}, a_{1}\right),\left(a_{4}, a_{3}, a_{1}, a_{2}\right)
\end{gathered}
$$

are also solutions of $P(x)=0$. Therefore, the most expected homotopy methods for this symmetric system are those which can get all isolated solutions through tracing only one homotopy path.

The solution number of $P(x)=0$ is exactly its Bézout number, hence applying any homotopy method without symmetry to solve $P(x)=0$, eight homotopy paths have to be traced, and consequently, they are all unsuitable to solve this system. This special symmetric structure also cannot be included in any type of symmetry mentioned in Section 1.4, and thus the mentioned symmetric homotopy methods cannot be applied to solve this polynomial system.

The hybrid homotopy constructed in [31] is as follows:

$$
H(x, t)=\gamma(1-t) Q(x)+t P(x)
$$

where

$$
Q(x)=\left(\begin{array}{c}
x_{1}+x_{2}+x_{3}+x_{4}+c_{1}^{0} \\
x_{1} x_{2}+x_{3} x_{4}+c_{2}^{0} \\
\left(s_{11} x_{1}+s_{12} x_{2}+s_{10}\right)\left(s_{21} x_{1}+s_{22} x_{2}+s_{20}\right) \\
\left(s_{31} x_{3}+s_{32} x_{4}+s_{30}\right)\left(s_{41} x_{3}+s_{42} x_{4}+s_{40}\right)
\end{array}\right)
$$

and $\gamma, c_{1}^{0}, c_{2}^{0}, s_{i j}$ 's are random nonzero complex numbers. The homotopy (2.5) and (2.6) is not symmetric, and eight homotopy paths have to be followed when applying the hybrid method to solve $P(x)=0$.

If we choose the symmetric hybrid homotopy in which $s_{i j}$ satisfies

$$
\left\{\begin{array} { l } 
{ s _ { 1 1 } = s _ { 2 2 } = s _ { 3 1 } = s _ { 4 2 } , } \\
{ s _ { 1 2 } = s _ { 2 1 } = s _ { 3 2 } = s _ { 4 1 } , } \\
{ s _ { 1 0 } = s _ { 2 0 } = s _ { 3 0 } = s _ { 4 0 } , }
\end{array} \quad \text { or } \quad \left\{\begin{array}{l}
s_{11}=s_{22}=s_{32}=s_{41}, \\
s_{12}=s_{21}=s_{31}=s_{42}, \\
s_{10}=s_{20}=s_{30}=s_{40},
\end{array}\right.\right.
$$

then the homotopy keeps the symmetry of the system (2.4), and when applying this symmetric homotopy to solve $P(x)=0$, only 1 homotopy path has to be traced. 
In particular, we can fix some coefficients in the start system, such as

$$
Q(x)=\left(\begin{array}{c}
p_{1}(x)+c_{1}^{0} \\
\cdots \\
p_{n+m}(x)+c_{n+m}^{0} \\
\left(x_{n+1}+x_{n+m+1} \mathbf{i}+s\right)\left(x_{n+1} \mathbf{i}+x_{n+m+1}+s\right) \\
\cdots \\
\left(x_{n+m}+x_{n+2 m} \mathbf{i}+s\right)\left(x_{n+m} \mathbf{i}+x_{n+2 m}+s\right)
\end{array}\right),
$$

where $s$ must be a random complex number instead of a fixed complex number, otherwise the homotopy will not satisfy accessibility.

2.2. Homotopy theorem and its proof. In order to find all isolated solutions of the target system through numerically tracing the solution paths of the homotopy map, we need to prove there exists a smooth path connecting every solution of the target system with one solution of the start system.

Theorem 2.1. $P(x), Q(x)$ are, respectively, as the systems in (1.5) and (2.2). There exists an open dense full-measure subset $U$ of $(n+m+4)$-dimensional complex space $\mathcal{C}^{n+m+4}$, such that for all $\left\{c_{1}^{0}, \ldots, c_{n+m}^{0}, s_{0}, s_{1}, s_{2}, \gamma\right\} \in U$ with $s_{1}^{2} \neq s_{2}^{2}$,

(1) solutions of $Q(x)=0$ are all isolated and nonsingular;

(2) the smoothness and accessibility properties hold for the homotopy map $H(x, t)$ defined in (2.1) and (2.2).

The following preliminary results are needed. A linear system $\mathcal{L}$ is a complex vector space

$$
\mathcal{L}=\left\{\sum_{i=1}^{r} k_{i} m_{i}(x):\left(k_{1}, \ldots, k_{r}\right) \in \mathcal{C}^{r}\right\}
$$

spanned by homogeneous polynomials $m_{1}(x), \ldots, m_{r}(x)$ of some fixed degree and parameterized by $\mathcal{C}^{r}$. Let $\mathcal{C} \mathcal{P}^{n}$ denote the $n$-dimensional complex projective space. The base locus of $\mathcal{L}$ is the set

$$
\left\{x \in \mathcal{C} \mathcal{P}^{n}: m_{i}(x)=0, i=1, \ldots, r\right\} .
$$

Lemma 2.2 (Bertini's Theorem [3]). Let $\mathcal{Z}$ be a nonsingular quasiprojective subvariety of $\mathcal{C P}^{n}$ of dimension $l$. Let $\mathcal{L}$ be a linear system as in (2.8). Then there exists a nonzero homogeneous polynomial $G$ in $r$ variables such that $G\left(k_{1}, \ldots, k_{r}\right) \neq 0$ implies that the intersection of $\mathcal{Z}$ with the hypersurface

$$
\sum_{i=1}^{r} k_{i} m_{i}(x)=0
$$

is of dimension $l-1$ and is nonsingular at each point not in the base locus of $\mathcal{L}$.

To ensure the process of path following can be started, we must show that from every solution of the start system, a homotopy path can be generated, that is, the solutions of the start system should be nonsingular.

Lemma 2.3. There exists an open dense full-measure subset $W_{1}$ of $\mathcal{C}^{n+m}$, such that for $\left(c_{1}^{0}, \ldots, c_{n+m}^{0}\right) \in W_{1}$ and any nonzero complex numbers $s_{0}, s_{1}$ and $s_{2}$ with $s_{1}^{2} \neq s_{2}^{2}$,

$$
s_{1} x_{n+i}+s_{2} x_{n+m+i}+s_{0}, s_{2} x_{n+i}+s_{1} x_{n+m+i}+s_{0}, 1 \leq j \leq m-1,
$$

are not both zero at any zero point of $Q(x)$ in (2.2). 
Proof. Without loss of generality, we prove the result for $i=m$ under the assumption that

$$
s_{1} x_{n+j}+s_{2} x_{n+m+j}+s_{0}, s_{2} x_{n+j}+s_{1} x_{n+m+j}+s_{0}, 1 \leq j \leq m-1,
$$

$(1 \leq j, m-1)$ are not both zero at any zero point of $Q(x)$.

Consider the following polynomial system

$$
R(x)=\left(\begin{array}{c}
p_{1}(x)+c_{1}^{0} \\
\cdots \\
p_{n+m}(x)+c_{n+m}^{0} \\
\cdots \\
\left(s_{1} x_{n+1}+s_{2} x_{n+m+1}+s_{0}\right)\left(s_{2} x_{n+1}+s_{1} x_{n+m+1}+s_{0}\right) \\
\left(s_{1} x_{n+m-1}+s_{2} x_{n+2 m-1}+s_{0}\right)\left(s_{2} x_{n+m-1}+s_{1} x_{n+2 m-1}+s_{0}\right) \\
s_{1} x_{n+m}+s_{2} x_{n+2 m}+s_{0} \\
s_{1} x_{n+2 m}+s_{2} x_{n+m}+s_{0}
\end{array}\right) .
$$

The Jacobian of

$$
R_{L}(x)=\left(\begin{array}{c}
\left(s_{1} x_{n+1}+s_{2} x_{n+m+1}+s_{0}\right)\left(s_{2} x_{n+1}+s_{1} x_{n+m+1}+s_{0}\right) \\
\cdots \\
\left(s_{1} x_{n+m-1}+s_{2} x_{n+2 m-1}+s_{0}\right)\left(s_{2} x_{n+m-1}+s_{1} x_{n+2 m-1}+s_{0}\right) \\
s_{1} x_{n+m}+s_{2} x_{n+2 m}+s_{0} \\
s_{1} x_{n+2 m}+s_{2} x_{n+m}+s_{0}
\end{array}\right)
$$

with respect to $x_{n+1}, \ldots, x_{n+2 m}$ is

$$
A=\left(\begin{array}{cccccccc}
a_{1} & & & 0 & a_{m+1} & & & \\
& \ddots & & 0 & & \ddots & & \\
& & a_{m-1} & 0 & & & a_{2 m-1} & \\
0 & \ldots & 0 & s_{1} & 0 & \ldots & 0 & s_{2} \\
0 & \ldots & 0 & s_{2} & 0 & \ldots & 0 & s_{1}
\end{array}\right),
$$

where

$$
\left\{\begin{array}{l}
a_{i}=s_{1}\left(s_{2} x_{n+i}+s_{1} x_{n+m+i}+s_{0}\right)+s_{2}\left(s_{1} x_{n+i}+s_{2} x_{n+m+i}+s_{0}\right), \\
a_{m+i}=s_{2}\left(s_{2} x_{n+i}+s_{1} x_{n+m+i}+s_{0}\right)+s_{1}\left(s_{1} x_{n+i}+s_{2} x_{n+m+i}+s_{0}\right),
\end{array}\right.
$$

for $1 \leq i \leq m-1$. Let $V\left(f_{1}, \ldots, f_{n}\right)$ denote the variety defined by $f_{1}, \ldots, f_{n}$. Because $s_{1}^{2} \neq s_{2}^{2}$, and one polynomial of

$$
s_{1} x_{n+i}+s_{2} x_{n+m+i}+s_{0}, s_{2} x_{n+i}+s_{1} x_{n+m+i}+s_{0}
$$

is nonzero for any solution of $Q(x)=0$, the matrix $A$ is of full rank and $\mathcal{Z}_{0}=$ $\mathcal{C}^{n+2 m} \cap V\left(R_{L}\right)$ is a nonsingular $(n+m-1)$-dimensional variety.

Let $\widetilde{R}(\widetilde{x})$, where $\widetilde{x}=\left(x_{0}, \ldots, x_{n+2 m}\right)$, be the homogenization of $R(x)$. Define

$$
g_{i}(\widetilde{x})=b_{i} \widetilde{p}_{i}(\widetilde{x})+c_{i} x_{0}^{d_{i}}
$$

for $i=1, \ldots, n+m$, where $\widetilde{p}_{i}(\widetilde{x})$ is the homogenization of $p_{i}(x)$, and each $g_{i}(\widetilde{x})$ is a homogeneous polynomial of degree $d_{i}=\operatorname{deg}\left(p_{i}\right)$.

The base locus of the linear system

$$
\mathcal{L}_{1}=\left\{b_{1} \widetilde{p}_{1}(\widetilde{x})+c_{1} x_{0}^{d_{1}}: b_{1}, c_{1} \in \mathcal{C}\right\}
$$

is contained in $V\left(x_{0}\right)$. Hence, by Bertini's Theorem, there exists a nonzero homogeneous polynomial $f_{1}\left(b_{1}, c_{1}\right)$ such that $\mathcal{Z}_{1}=\mathcal{Z}_{0} \cap V\left(b_{1} \widetilde{p}_{1}(\widetilde{x})+c_{1} x_{0}^{d_{1}}\right)$ is a nonsingular $(n+m-2)$-dimensional quasiprojective variety for $f_{1}\left(b_{1}, c_{1}\right) \neq 0$. 
$f_{1}\left(b_{1}, c_{1}\right)$ is homogeneous, so the polynomial $\widehat{f}_{1}\left(c_{1}\right)=f_{1}\left(1, c_{1}\right)$ is not identically zero, and its solution set $M_{1}$ is a finite subset of $\mathcal{C}$. Therefore, for all $c_{1}^{0} \notin M_{1}$, $\mathcal{Z}_{0} \cap V\left(p_{1}(\widetilde{x})+c_{1}^{0} x_{0}^{d_{1}}\right)$ is a nonsingular $(n+m-2)$-dimensional quasiprojective variety, and hence $\mathcal{Z}_{1}=\mathcal{Z}_{0} \cap V\left(p_{1}(x)+c_{1}^{0}\right)$ is a nonsingular $(n+m-2)$-dimensional quasivariety.

By repeating the process $n+m-1$ times, there exist $n+m-1$ subsets $M_{1}, \ldots$, $M_{n+m-1}$ of $\mathcal{C}$, such that for $c_{i}^{0} \notin M_{i}$, the solution set $\mathfrak{N}$ to

$$
\left(\begin{array}{c}
p_{1}(x)+c_{1}^{0} \\
\cdots \\
p_{n+m-1}(x)+c_{n+m-1}^{0} \\
\cdots \\
\left(s_{1} x_{n+1}+s_{2} x_{n+m+1}+s_{0}\right)\left(s_{2} x_{n+1}+s_{1} x_{n+m+1}+s_{0}\right) \\
\left(s_{1} x_{n+m-1}+s_{2} x_{n+2 m-1}+s_{0}\right)\left(s_{2} x_{n+2 m-1}+s_{1} x_{n+m-1}+s_{0}\right) \\
s_{1} x_{n+m}+s_{2} x_{n+2 m}+s_{0} \\
s_{2} x_{n+2 m}+s_{1} x_{n+m}+s_{0}
\end{array}\right)=0
$$

is of 0 dimension and the set $M_{n+m}=\left\{c_{n+m}^{0}=-p_{n+m}(x): x \in \mathfrak{N}\right\}$ is a finite set in $\mathcal{C}$, and for $\left(c_{1}^{0}, \ldots, c_{n+m}^{0}\right)$ chosen from $W_{1}=\mathcal{C}^{n+m} \backslash\left(M_{1} \times \cdots \times M_{n+m}\right)$, an open dense full-measure subset of $\mathcal{C}^{n+m}, R(x)=0$ has no solution. Thus the lemma follows.

Lemma 2.4. There exists an open dense full-measure subset $W_{2}$ of $\mathcal{C}^{n+m}$, such that for $\left(c_{1}^{0}, \ldots, c_{n+m}^{0}\right) \in W_{2}$ and any nonzero complex numbers $s_{0}, s_{1}, s_{2}$ with $s_{1}^{2} \neq s_{2}^{2}$, the solution set of $Q(x)=0$ in (2.2) consists of isolated nonsingular points.

Proof. Let $\mathcal{Y} \subset \mathcal{C}^{n+2 m}$ be the solution set to

$$
\left(\begin{array}{c}
\left(s_{1} x_{n+1}+s_{2} x_{n+m+1}+s_{0}\right)\left(s_{2} x_{n+1}+s_{1} x_{n+m+1}+s_{0}\right) \\
\ldots \\
\left(s_{1} x_{n+m}+s_{2} x_{n+2 m}+s_{0}\right)\left(s_{2} x_{n+m}+s_{1} x_{n+2 m}+s_{0}\right)
\end{array}\right)=0 .
$$

The Jacobian of the system (2.10) with respect to $x_{n+1}, \ldots, x_{n+2 m}$ is

$$
B=\left(\begin{array}{cccccc}
a_{1} & & & a_{m+1} & & \\
& \ddots & & & \ddots & a_{2 m}
\end{array}\right),
$$

where $a_{i}$ 's are as in (2.9) for $i=1, \ldots, m$.

By Lemma 2.3. there exists a full-measure subset $W_{1}$ of $\mathcal{C}^{n+m}$ such that for $\left(c_{1}^{0}, \ldots, c_{n+m}^{0}\right) \in W_{1}$ and any nonzero complex numbers $s_{0}, s_{1}, s_{2}$ with $s_{1}^{2} \neq s_{2}^{2}$, only one equation of

$$
s_{1} x_{n+k}+s_{2} x_{n+m+k}+s_{0}=0, s_{2} x_{n+k}+s_{1} x_{n+m+k}+s_{0}=0
$$

holds for any solution of $Q(x)=0$, thus $\operatorname{rank}_{\mathcal{C}} B=m$ and $\mathcal{Y}$ is a nonsingular $(n+m)$-dimensional variety.

After repeatedly utilizing Bertini's Theorem $n+m$ times, there exists a fullmeasure subset $W_{2} \subset W_{1}$ of $\mathcal{C}^{n+m}$ such that for $\left(c_{1}^{0}, \ldots, c_{n+m}^{0}\right) \in W_{2}$ and any nonzero complex numbers $s_{0}, s_{1}, s_{2}$ with $s_{1}^{2} \neq s_{2}^{2}$, all solutions to $Q(x)=0$ are isolated and nonsingular.

Let $\left\langle f_{1}, \ldots, f_{n}\right\rangle$ denote the homogeneous ideal generated by homogeneous polynomials $f_{1}, \ldots, f_{n},\left\langle f_{1}, \ldots, f_{n}\right\rangle_{M}$ denote the subset of $\left\langle f_{1}, \ldots, f_{n}\right\rangle$ consisting of degree $M$ polynomials. 
Lemma $2.5(9)$. Let $f_{1}, \ldots, f_{n}$ be homogeneous polynomials in the variables $x_{0}, \ldots, x_{n}$ such that the set $S=\left\{\left(x_{0}, \ldots, x_{n}\right) \in V\left(f_{1}, \ldots, f_{n}\right): x_{0} \neq 0\right\}$ consists of isolated points. Then there exists a positive integer $K_{0}$ such that for $K \geq K_{0}$,

$$
\left\langle f_{1}, \ldots, f_{n}, x_{0}^{K_{0}}\right\rangle_{M}=\left\langle f_{1}, \ldots, f_{n}, x_{0}^{K}\right\rangle_{M}
$$

for sufficiently large integers $M$.

We need to prove a path generating from a solution of the homotopy map at $t=1$ and extending backward to $t=0$ will not tend to infinity. The key lemma to detect paths diverging to infinity is the following.

Lemma 2.6 ([10]). Let $H: \mathcal{C}^{n} \times T \rightarrow \mathcal{C}^{n}$, where $T \subset \mathcal{R}$ is an open subset, $H$ is continuous in $t$ and, for each $t, h_{i}(x, t)$ is a polynomial in $x$ whose homogenization is denoted by $\widetilde{h}_{i}$. Suppose there is an integer $K<m$ such that $\operatorname{dim}_{\mathcal{C}}\left\langle\widetilde{h}_{1}, \ldots, \widetilde{h}_{n}, x_{0}{ }^{k}\right\rangle_{m}$ is independent of $t \in T$ and $k \in\{K, K+1\}$. Then no path of solutions of $H(x, t)=0$ parameterized by $t$ tends to infinity as $t \rightarrow t_{0} \in T$.

Lemma 2.7 ([2]). If $F_{0}, \ldots, F_{n}$ are homogeneous polynomials in $x_{0}, \ldots, x_{n}$ of degrees $d_{0}, \ldots, d_{n}$, then $F_{0}=\cdots=F_{n}=0$ has a nontrivial solution if and only if $\operatorname{Res}\left(F_{0}, \ldots, F_{n}\right)=0$, here, $\operatorname{Res}\left(F_{0}, \ldots, F_{n}\right)$ is the resultant of $F_{0}, \ldots, F_{n}$, a homogeneous polynomial in all possible coefficients of $F_{i}(i=0, \ldots, n)$.

Proof of Theorem 2.1. (1). Rewrite $H(x, t)=0$ as $H(x, \tau)=Q(x)+\tau P(x)=0$, where $\tau=\frac{t}{\gamma(1-t)}$, and let $\widetilde{H}(\widetilde{x}, \tau)=\widetilde{Q}(\widetilde{x})+\tau \widetilde{P}(\widetilde{x})$ be the homogenization of $H(x, \tau)$, where $\widetilde{Q}(\widetilde{x})$ and $\widetilde{P}(\widetilde{x})$ are the homogenization of $Q(x)$ and $P(x)$.

Denote $\left(\frac{\partial \widetilde{H}(\widetilde{x}, \tau)}{\partial \widetilde{x}}\right)$ by $\widetilde{H}^{\prime}(\widetilde{x}, \tau)$. If the solutions to $\widetilde{H}(\widetilde{x}, \tau)=0$ are singular, then the following system

$$
\left(\begin{array}{c}
\operatorname{det}\left(\widetilde{H^{\prime}}(\widetilde{x}, \tau)\right) \\
\widetilde{H}(\widetilde{x}, \tau)
\end{array}\right)=0
$$

has nontrivial solutions, thus by Lemma 2.7 the resultant $\operatorname{Res}\left(\widetilde{H}, \operatorname{det}\left(\widetilde{H}^{\prime}\right)\right)$, denoted by $r(\tau)$, of $\widetilde{H}$ and $\operatorname{det}\left(\widetilde{H}^{\prime}\right)$ equals 0 . Since solutions to $Q(x)=0$ are regular, $r(0) \neq 0$ and $r(\tau)$ is not identically zero, the solution set $S_{1}$ to $r(\tau)=0$ is finite, and the complementary set $S_{2}$ of $\left\{\gamma \in \mathcal{C}: \frac{t}{\gamma(1-t)} \in S_{1}\right\}$ is an open dense full-measure subset of $\mathcal{C}$. For $\gamma \in S_{2}, r(\tau) \neq 0$, and thus (2.11) has no solutions, that is, there exists an open dense full-measure subset $U_{1}$ of $\mathcal{C}^{n+m+4}$ such that the solutions to $H(x, t)=0$ are nonsingular for $\left(c_{1}^{0}, \ldots, c_{n+m}^{0}, s_{0}, s_{1}, s_{2}, \gamma\right) \in U_{1}$ with $s_{1}^{2} \neq s_{2}^{2}$.

We first show that there exists an open dense full-measure subset $W_{3}$ of $\mathcal{C}^{n+m}$ such that $W_{3} \subset W_{1}$, and for any $\left(c_{1}^{0}, \ldots, c_{n+m}^{0}\right) \in W_{3}$, and $s_{0}, s_{1}, s_{2}$ with $s_{1}^{2} \neq s_{2}^{2}$, the Jacobian of the system

$$
S(x)=\left(\begin{array}{c}
q_{n+m+1}(x)+\tau\left(x_{n+1}^{2}+x_{n+m+1}^{2}-1\right) \\
\cdots \\
q_{n+2 m}(x)+\tau\left(x_{n+m}^{2}+x_{n+2 m}^{2}-1\right)
\end{array}\right)=0
$$

where

$$
q_{n+m+i}(x)=\left(s_{1} x_{n+i}+s_{2} x_{n+m+i}+s_{0}\right)\left(s_{2} x_{n+i}+s_{1} x_{n+m+i}+s_{0}\right)
$$

is the $(n+m+i)$-th equation of the start system $Q(x)=0$ for all $i=1, \ldots, m$, is full-rank at solutions of $H(x, \tau)=0$. 
The Jacobian of $S(x)=0$ with respect to $x_{n+1}, \ldots, x_{n+2 m}$ is

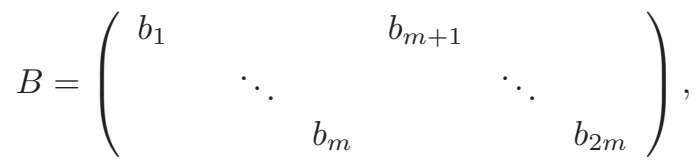

where

$\left\{\begin{array}{l}b_{i}=2 \tau x_{n+i}+\left(s_{1}\left(s_{2} x_{n+i}+s_{1} x_{n+m+i}+s_{0}\right)+s_{2}\left(s_{1} x_{n+i}+s_{2} x_{n+m+i}+s_{0}\right)\right), \\ b_{m+i}=2 \tau x_{n+m+i}+\left(s_{1}\left(s_{1} x_{n+i}+s_{2} x_{n+m+i}+s_{0}\right)+s_{2}\left(s_{2} x_{n+i}+s_{1} x_{n+m+i}+s_{0}\right)\right),\end{array}\right.$ for $i=1, \ldots, m$.

In the following, we will prove $b_{i}$ and $b_{m+i}$ are not both zero at solutions of $H(x, \tau)=0$, that is, the following system

$$
T(x)=\left(\begin{array}{c}
b_{i} \\
b_{m+i} \\
h_{n+m+i}^{\tau}
\end{array}\right)=0
$$

where $h_{n+m+i}^{\tau}$ is the $(n+m+i)$-th polynomial in $H(x, \tau)$, does not hold at solutions of $H(x, \tau)=0$.

Suppose $T(x)=0$ holds at a solution point of $H(x, \tau)=0$, let

$$
\widetilde{T}(\widetilde{x})=\left(\begin{array}{c}
\widetilde{b}_{i} \\
\widetilde{b}_{m+i} \\
\widetilde{h}_{n+m+i}^{s}
\end{array}\right)
$$

be the homogenization of $T(x)$, then from Lemma 2.7 the resultant of $\widetilde{b}_{i}, \widetilde{b}_{m+i}$, $\widetilde{h}_{n+m+i}^{s}$, denoted by $r_{1}(\tau)$, equals zero.

When $\tau=0, T(x)$ in (2.13) is as follows:

$$
\left(\begin{array}{c}
s_{1}\left(s_{2} x_{n+i}+s_{1} x_{n+m+i}+s_{0}\right)+s_{2}\left(s_{1} x_{n+i}+s_{2} x_{n+m+i}+s_{0}\right) \\
s_{1}\left(s_{1} x_{n+i}+s_{2} x_{n+m+i}+s_{0}\right)+s_{2}\left(s_{2} x_{n+i}+s_{1} x_{n+m+i}+s_{0}\right. \\
\left(s_{2} x_{n+i}+s_{1} x_{n+m+i}+s_{0}\right)\left(s_{1} x_{n+i}+s_{2} x_{n+m+i}+s_{0}\right)
\end{array}\right) .
$$

From the third equation, we can see that at least one of polynomials of

$$
s_{2} x_{n+i}+s_{1} x_{n+m+i}+s_{0}, s_{1} x_{n+i}+s_{2} x_{n+m+i}+s_{0}
$$

is zero at the solution to $H(x, 0)=0$. Because $s_{1}^{2} \neq s_{2}^{2}, s_{1}$ and $s_{2}$ are not zeroes. Then from the first and second equation, we can see that if one polynomial in (2.14) is zero at the solution to $H(x, 0)=0$, then the other is also zero, which is inconsistent with Lemma 2.3. Hence $T(x)=0$ is not zero at the solution to $H(x, 0)=0$, that is, for $\left(c_{1}^{0}, \ldots, c_{n+m}^{0}\right) \in W_{1}, T(x)$ is not zero at the solution of $H(x, t)=0$ when $\tau=0$.

From Lemma 2.7, we can get $r_{1}(0) \neq 0$, thus $r_{1}(\tau)$ is not identically zero, and its solution $N_{1}$ is a finite subset of $\mathcal{C}$. The complementary set $D$ of $\left\{\gamma \in \mathcal{C}: \frac{t}{\gamma(1-t)} \in\right.$ $\left.N_{1}\right\}$ is an open dense full-measure subset of $\mathcal{C}$, and for $\gamma \in D, r(\tau) \neq 0$, and thus (2.13) has no solution, i.e., $b_{i}$ and $b_{m+i}$ are not both zero, thus $\operatorname{rank}_{\mathcal{C}} B=m$.

By the same argument as the proof of Lemma 2.3. we can know that for any $s_{0}$, $s_{1}$ and $s_{2}$ with $s_{1}^{2} \neq s_{2}^{2}$, there exists a full-measure subset $W_{3}$ of $\mathcal{C}^{n+m}$ such that $W_{3} \subset W_{1}$ for $\left(c_{1}^{0}, \ldots, c_{n+m}^{0}\right) \in W_{3}$, all solutions to $H(x, t)=0$ are isolated and nonsingular.

(2) Denote by $S_{d}$ the complex vector space of degree $d$ homogeneous polynomials in $x_{0}, \ldots, x_{n+2 m}$ with complex coefficients. Let $h_{i}$ be the $i$-th polynomial 
of $H(x, t)$, and $\widetilde{h}_{1}, \ldots, \widetilde{h}_{n+2 m}$ the homogenization of $h_{1}, \ldots, h_{n+2 m}, \widetilde{h}_{i} \in S_{d_{i}}$. For $d \geq \max \left\{d_{i}\right\}$, define the linear transformation

$$
\begin{gathered}
T_{d}\left(\widetilde{h}_{1}, \ldots, \widetilde{h}_{n+2 m}, x_{0}^{K}\right): S_{d-d_{1}} \times \cdots \times S_{d-d_{n+2 m}} \times S_{d-K} \rightarrow S_{d}, \\
T_{d}\left(\widetilde{h}_{1}, \ldots, \widetilde{h}_{n+2 m}, x_{0}^{K}\right)\left(r_{1}, \ldots, r_{n+2 m+1}\right)=\widetilde{h}_{1} r_{1}+\cdots+\widetilde{h}_{n+2 m} r_{n+2 m}+x_{0}^{K} r_{n+2 m+1} .
\end{gathered}
$$

Let $\mathfrak{T}_{d}$ be the matrix representation of $T_{d}$ using the basis of all degree $d$ monomials for each $S_{d}$. It follows that

$$
\operatorname{rank}_{\mathcal{C}} \mathfrak{T}_{d}\left(\widetilde{h}_{1}, \ldots, \widetilde{h}_{n+2 m}, x_{0}^{K}\right)=\operatorname{dim}_{\mathcal{C}}\left\langle\widetilde{h}_{1}, \ldots, \widetilde{h}_{n+2 m}, x_{0}^{K}\right\rangle_{d}
$$

The solutions to $Q(x)=0$ are all isolated and nonsingular, thus by Lemma 2.5. there exist integers $K$ and $e_{0}\left(e_{0}>K\right)$ such that for $e \geq e_{0}$,

$$
\left\langle\widetilde{h}_{1}, \ldots, \widetilde{h}_{n+2 m}, x_{0}{ }^{K}\right\rangle_{e}=\left\langle\widetilde{h}_{1}, \ldots, \widetilde{h}_{n+2 m}, x_{0}{ }^{K+1}\right\rangle_{e}
$$

holds for $t=0$.

Let $C$ be the highest-rank subset of $\mathfrak{T}_{e}\left(\widetilde{h}_{1}, \ldots, \widetilde{h}_{n+2 m}, x_{0}^{K}\right)(K<e)$, and it satisfies the following condition: $\operatorname{det}(C)=D\left(c_{1}^{0}, \ldots, c_{n+m}^{0}, s_{0}, s_{1}, s_{2}, \gamma, t\right)$ is not identically zero as a polynomial. The determinant of the matrix $C \operatorname{det}(C)$, if considered as a polynomial in the variables $c_{1}^{0}, \ldots, c_{n+m}^{0}, s_{0}, s_{1}, s_{2}, \gamma$, has the leading monomial

$$
b(1-t)^{\theta_{0}}\left(c_{1}^{0}\right)^{\theta_{1}} \cdots\left(c_{n+m}^{0}\right)^{\theta_{n+m}} s_{0}{ }^{\theta_{n+m+1}} s_{1}{ }^{\theta_{n+m+2}} s_{2}{ }^{\theta_{n+m+3}} \gamma^{\theta_{n+m+4}}
$$

for some nonnegative integers $\theta_{1}, \ldots, \theta_{n+m+4}$ and $b \in \mathcal{C}^{*}$, so $\operatorname{det}(C)$ is not identically zero for each $t_{0} \neq 1$ and vanishes on a complex codimension 1 subset $\mathcal{Z}_{t_{0}}$ of $\mathcal{C}^{n+m+4}$. The union $\mathcal{Z}=\bigcup_{t_{0} \in \mathcal{R} \backslash\{1\}} \mathcal{Z}_{t_{0}}$ of $\mathcal{Z}_{t_{0}}$ is of real codimension 1 in $\mathcal{C}^{n+m+4}$, and hence the complementary set $U_{2}$ of the closure is an open dense full-measure subset of $\mathcal{C}^{n+m+4}$. For all $\left(c_{1}^{0}, \ldots, c_{n+m}^{0}, s_{0}, s_{1}, s_{2}, \gamma\right) \in U_{2}$, $\operatorname{rank}_{\mathcal{C}} \mathfrak{T}_{e}\left(\widetilde{h}_{1}, \ldots, \widetilde{h}_{n+2 m}, x_{0}^{K}\right)$ is independent of $t \in \mathcal{R} \backslash\{1\}$. Since the equation (2.15) holds, $\operatorname{dim}_{\mathcal{C}}\left\langle\widetilde{h}_{1}, \ldots, \widetilde{h}_{n+2 m}, x_{0}{ }^{K}\right\rangle_{e}$ is independent of $t \in \mathcal{R} \backslash\{1\}$.

Replacing $K$ by $K+1$ in the above, the same argument holds for $K+1$ on an open dense full-measure subset $U_{3}$ of $\mathcal{C}^{n+m+4}$.

From (2.16), we can see that

$$
\operatorname{dim}_{\mathcal{C}}\left\langle\widetilde{h}_{1}, \ldots, \widetilde{h}_{n+2 m}, x_{0}{ }^{K+1}\right\rangle_{e}=\operatorname{dim}_{\mathcal{C}}\left\langle\widetilde{h}_{1}, \ldots, \widetilde{h}_{n+2 m}, x_{0}{ }^{K}\right\rangle_{e}
$$

holds for $t=0$. Because $\operatorname{dim}_{\mathcal{C}}\left\langle\widetilde{h}_{1}, \ldots, \widetilde{h}_{n+2 m}, x_{0}{ }^{K}\right\rangle_{e}$ is independent of $t \in \mathcal{R} \backslash\{1\}$,

$$
\operatorname{dim}_{\mathcal{C}}\left\langle\widetilde{h}_{1}, \ldots, \widetilde{h}_{n+2 m}, x_{0}{ }^{K+1}\right\rangle_{e}=\operatorname{dim}_{\mathcal{C}}\left\langle\widetilde{h}_{1}, \ldots, \widetilde{h}_{n+2 m}, x_{0}{ }^{K}\right\rangle_{e}
$$

holds for all $t \in \mathcal{R} \backslash\{1\}$. Therefore, for all $\left(c_{1}^{0}, \ldots, c_{n+m}^{0}, s_{0}, s_{1}, s_{2}, \gamma\right) \in\left(U_{2} \cap U_{3}\right)$, $\operatorname{dim}_{\mathcal{C}}\left\langle\widetilde{h}_{1}, \ldots, \widetilde{h}_{n+2 m}, x_{0}{ }^{k}\right\rangle_{e}$ is independent of $t \in \mathcal{R} \backslash\{1\}$ and $k \in\{K, K+1\}$. By Lemma 2.6. all homotopy paths remain finite for $t \neq 1$. Thus the accessibility follows for all $\left(c_{1}^{0}, \ldots, c_{n+m}^{0}, s_{0}, s_{1}, s_{2}, \gamma\right) \in U=U_{1} \cap U_{2} \cap U_{3}$ with $s_{1}^{2} \neq s_{2}^{2}$.

\section{The SOlution PROCEDURE}

Applying the symmetric homotopy (2.1) and (2.2), and combining the homotopy method with symbolic methods, such as decomposition, variable substitution and some reduction techniques, we present a symmetric hybrid method to solve polynomial systems transformed from MTPS's.

Algorithm 3.1 (Symmetric hybrid method).

Step 1. Construct the symmetric hybrid homotopy as in (2.1) and (2.2). 
Step 2. Decompose the start system in (2.2) into $2^{m}$ subsystems $Q_{\sigma}(x)=0$ as (2.3).

Step 3. By variable substitution

$$
x_{n+m+i}=l_{i}\left(x_{n+i}\right):=-\frac{s_{\sigma_{i}}}{s_{3-\sigma_{i}}} x_{n+i}-\frac{s_{0}}{s_{3-\sigma_{i}}}
$$

for $i=1, \ldots, m, Q_{\sigma}(x)=0$ can be transformed to a new simpler system $\widehat{Q}_{\sigma}(\widehat{x})=0$ with less variables and equations:

$$
\widehat{Q}_{\sigma}(\widehat{x})=\left(\begin{array}{c}
q_{1}(\widehat{x})+c_{1}^{0} \\
\cdots \\
q_{n+m}(\widehat{x})+c_{n+m}^{0}
\end{array}\right),
$$

where $\widehat{x}=\left(x_{1}, \ldots, x_{n+m}\right)$ and for all $1 \leq i \leq n+m$,

$$
q_{i}(\widehat{x})=\sum_{\alpha \in S_{i}} b_{i \alpha}\left(x_{1}, \ldots, x_{n+m}, l_{1}\left(x_{n+1}\right), \ldots, l_{m}\left(x_{n+m}\right)\right)^{\alpha} .
$$

Hence solving $Q(x)=0$ is equivalent to solving $2^{m}$ systems $\widehat{Q}_{\sigma}(\widehat{x})=0$ of dimension $n+m$.

Step 4. Apply Algorithm 3.2 to reduce the subsystems so that they will have lower upper bounds of solution number, and even assert some of them have no solutions indeed.

Step 5. Solve the reduced subsystems by some existing homotopy methods. Note that if the target system, and hence the start system, is symmetric, it is not necessary to solve all the reduced subsystems. Only the subsystems with representative solutions should be solved.

Step 6. Expand the solutions $x^{*}=\left(x_{1}^{*}, \ldots, x_{n+m}^{*}\right)$ of all subsystems by (3.1) to get solutions $x^{*}=\left(x_{1}^{*}, \ldots, x_{n+2 m}^{*}\right)$ to $Q(x)=0$. These solutions serve as the start points of the homotopy in (2.1).

Step 7. Trace homotopy paths. Applying predictor-corrector method to follow solution paths of (2.1) to get all isolated solutions of the target system $P(x)=0$.

The details of the symbolic tricks applied in Algorithm 3.1 are described in the following algorithm.

Algorithm 3.2 (Polynomial system reduction). Given the polynomial system $P(x)$ $=\left(p_{1}(x), \ldots, p_{n}(x)\right)^{\top}=0$, where $x \in \mathcal{C}^{n}$.

Step 1. Using lexicographic order with $x_{1}>x_{2}>\cdots>x_{n}$, we form a vector $v=\left(v_{1}, \ldots, v_{N}\right)^{\top}$, which consists of all monomials in $P(x)$ and such that $v_{i}>v_{k}$ for $i<k$, let $c$ be the vector consisting of all constant terms of $P(x)=0$, then a matrix $A$, such that $A x+c=0$, can be constructed.

Step 2. For $i=1, \ldots, N$ :

1. Find the element having the maximum absolute value in the $i$-th column as the pivot.

2. Eliminate the other elements in this column by this pivot.

Step 3. If the elimination matrix is rank-deficient, then we can assert that this system has no solution. Otherwise, output the reduced system. 
An important consideration of our algorithm is on parallel implementation. Homotopy methods are naturally parallel because the solution paths of a homotopy can all be followed independently. For our method, in the stage of solving all subsystems, we can apply the polyhedral homotopy method to solve a system which has the same monomial structure as the subsystems and random coefficients, then apply the coefficient-parameter homotopy method to solve all subsystems. In the stage of applying the homotopy method to solve the target system, the coefficientparameter homotopy method is applied again. All of this work is mainly performing path tracking, therefore, we can assign these jobs to different processors, and furthermore, our methods can scale very well due to the low communication overhead in the process of path tracking. The parallel implementation of our symmetric hybrid method is still developing.

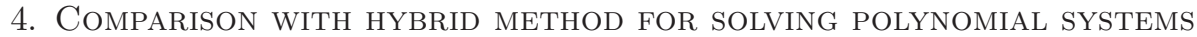

In this section, we state the difference of our symmetric hybrid method with the hybrid method in 31, and also declare the advantages of our algorithms in solving the polynomial systems coming from MTPS's.

Similar to the hybrid method in 31, the start system can be decomposed to some subsystems in $n+m$ variables, so it also possesses the advantage of the hybrid method in 31. On the other hand, due to the application of the symmetry, the symmetric hybrid method has two additional advantages as follows:

- In Step 5, if the reduced subsystems are symmetric, exploiting homotopy methods for symmetric polynomial systems, only the representative paths should be traced, and hence decreases the computational work considerably. In Step 7, the same advantage holds if the target system is symmetric.

- The symmetric hybrid method can keep the sparse structure of the target system better than the hybrid method in 31. due to the symmetry of the last $m$ equations in the start system. The subsystems can be considerably reduced in Bézout number and mixed volume even when the target system can hardly be reduced. More importantly, at some extreme cases, we can find that part of the reduced subsystems $\widehat{Q}_{\sigma}(\widehat{x})=0$ need not be solved because some equations in them are obviously inconsistent. We name this class of subsystems inconsistent systems and other subsystems consistent systems. Thus the computational work can be reduced considerably, as shown in the following example.

Example 4.1 (Example 1.2 continued). For the transformed polynomial system in (1.7) when $n=3$, we construct the start system as that in 31]:

$$
Q(x)=\left(\begin{array}{c}
x_{1}+x_{2}+x_{3}-c_{1}^{0} \\
x_{1}^{2}+x_{2}^{2}+x_{3}^{2}-c_{2}^{0} \\
x_{1} x_{4}+x_{2} x_{5}+x_{3} x_{6}-c_{3}^{0} \\
x_{1} x_{7}+x_{2} x_{8}+x_{3} x_{9}-c_{4}^{0} \\
x_{1} x_{4}^{2}+x_{2} x_{5}^{2}+x_{3} x_{6}^{2}-c_{5}^{0} \\
x_{1} x_{4} x_{7}+x_{2} x_{5} x_{8}+x_{3} x_{6} x_{9}-c_{6}^{0} \\
\left(a_{11}^{1} x_{4}+a_{12}^{1} x_{7}+a_{10}^{1}\right)\left(a_{11}^{2} x_{4}+a_{12}^{2} x_{7}+a_{10}^{2}\right) \\
\left(a_{21}^{1} x_{5}+a_{22}^{1} x_{8}+a_{20}^{1}\right)\left(a_{21}^{2} x_{5}+a_{22}^{2} x_{8}+a_{20}^{2}\right) \\
\left(a_{31}^{1} x_{6}+a_{32}^{1} x_{9}+a_{30}^{1}\right)\left(a_{31}^{2} x_{6}+a_{32}^{2} x_{9}+a_{30}^{2}\right)
\end{array}\right)=0 .
$$


$Q(x)=0$ can be decomposed into eight subsystems. For $\sigma=\left(\sigma_{1}, \sigma_{2}, \sigma_{3}\right)$, where $\sigma_{i} \in\{1,2\}$,

$$
Q_{\sigma}(x)=\left(\begin{array}{c}
x_{1}+x_{2}+x_{3}-c_{1}^{0} \\
x_{1}^{2}+x_{2}^{2}+x_{3}^{2}-c_{2}^{0} \\
x_{1} x_{4}+x_{2} x_{5}+x_{3} x_{6}-c_{3}^{0} \\
x_{1} x_{7}+x_{2} x_{8}+x_{3} x_{9}-c_{4}^{0} \\
x_{1} x_{4}^{2}+x_{2} x_{5}^{2}+x_{3} x_{6}^{2}-c_{5}^{0} \\
x_{1} x_{4} x_{7}+x_{2} x_{5} x_{8}+x_{3} x_{6} x_{9}-c_{6}^{0} \\
a_{11}^{\sigma_{1}} x_{4}+a_{12}^{\sigma_{1}} x_{7}+a_{10}^{\sigma_{1}} \\
a_{21}^{\sigma_{2}} x_{5}+a_{22}^{\sigma_{2}} x_{8}+a_{20}^{\sigma_{2}} \\
a_{31}^{\sigma_{3}} x_{6}+a_{32}^{\sigma_{3}} x_{9}+a_{30}^{\sigma_{3}}
\end{array}\right)=0 .
$$

Eliminating $x_{7}, x_{8}, x_{9}$ and by some simple symbolic computation, such as variable substitution and collecting like terms, (4.1) is equivalently transformed to the system of the form

$$
\widehat{Q}_{\sigma}(\widehat{x})=\left(\begin{array}{c}
x_{1}+x_{2}+x_{3}-c_{1}^{0} \\
x_{1}^{2}+x_{2}^{2}+x_{3}^{2}-c_{2}^{0} \\
x_{1} x_{4}+x_{2} x_{5}+x_{3} x_{6}-c_{3}^{0} \\
x_{1} x_{4}^{2}+x_{2} x_{5}^{2}+x_{3} x_{6}^{2}-c_{5}^{0} \\
b_{1}^{1} x_{2} x_{5}+b_{2}^{1} x_{3} x_{6}+b_{3}^{1} x_{1}+b_{1}^{1} x_{2}+b_{5}^{1} x_{3}+b_{6}^{1} \\
b_{1}^{2} x_{2} x_{5}^{2}+b_{2}^{2} x_{3} x_{6}^{2}+b_{3}^{2} x_{1} x_{4}+b_{4}^{2} x_{2} x_{5}+b_{5}^{2} x_{3} x_{6}+b_{6}^{2}
\end{array}\right)=0
$$

whose mixed volume is 12 .

We construct the start system as that in the symmetric hybrid method:

$$
Q^{s}(x)=\left(\begin{array}{c}
x_{1}+x_{2}+x_{3}-c_{1}^{0} \\
x_{1}^{2}+x_{2}^{2}+x_{3}^{2}-c_{2}^{0} \\
x_{1} x_{4}+x_{2} x_{5}+x_{3} x_{6}-c_{3}^{0} \\
x_{1} x_{7}+x_{2} x_{8}+x_{3} x_{9}-c_{4}^{0} \\
x_{1} x_{4}^{2}+x_{2} x_{5}^{2}+x_{3} x_{6}^{2}-c_{5}^{0} \\
x_{1} x_{4} x_{7}+x_{2} x_{5} x_{8}+x_{3} x_{6} x_{9}-c_{6}^{0} \\
\left(x_{4}+x_{7} \mathbf{i}+s\right)\left(x_{4} \mathbf{i}+x_{7}+s\right) \\
\left(x_{5}+x_{8} \mathbf{i}+s\right)\left(x_{5} \mathbf{i}+x_{8}+s\right) \\
\left(x_{6}+x_{9} \mathbf{i}+s\right)\left(x_{6} \mathbf{i}+x_{9}+s\right)
\end{array}\right)=0 .
$$

$Q^{s}(x)=0$ can be decomposed into eight subsystems. For $\sigma=(1,2,2)$,

$$
Q_{\sigma}^{s}(x)=\left(\begin{array}{c}
x_{1}+x_{2}+x_{3}-c_{1}^{0} \\
x_{1}^{2}+x_{2}^{2}+x_{3}^{2}-c_{2}^{0} \\
x_{1} x_{4}+x_{2} x_{5}+x_{3} x_{6}-c_{3}^{0} \\
x_{1} x_{7}+x_{2} x_{8}+x_{3} x_{9}-c_{4}^{0} \\
x_{1} x_{4}^{2}+x_{2} x_{5}^{2}+x_{3} x_{6}^{2}-c_{5}^{0} \\
x_{1} x_{4} x_{7}+x_{2} x_{5} x_{8}+x_{3} x_{6} x_{9}-c_{6}^{0} \\
x_{4}+x_{7} \mathbf{i}+s \\
x_{5} \mathbf{i}+x_{8}+s \\
x_{6} \mathbf{i}+x_{9}+s
\end{array}\right)=0 .
$$


Eliminating $x_{7}, x_{8}, x_{9}$ and by some simple symbolic computation, e.g., polynomial addition and variable substitution, (4.2) is equivalently transformed to

$$
\widehat{Q}_{\sigma}^{s}(\widehat{x})=\left(\begin{array}{c}
x_{1}+x_{2}+x_{3}-c_{1}^{0} \\
x_{1}^{2}+x_{2}^{2}+x_{3}^{2}-c_{2}^{0} \\
2 x_{1} x_{4}+(s-s \mathbf{i}) x_{1}+s c_{1}^{0} \mathbf{i}+c_{4}^{0} \mathbf{i}-c_{3}^{0} \\
2 x_{2} x_{5}+2 x_{3} x_{6}+(s-s \mathbf{i})\left(x_{2}+x_{3}\right)-s c_{1}^{0}-c_{4}^{0} \mathbf{i}-c_{3}^{0} \\
2 x_{1} x_{4}^{2}+(s-s \mathbf{i}) x_{1} x_{4}+c_{3}^{0} s \mathbf{i}+c_{6}^{0} \mathbf{i}-c_{5}^{0} \\
2 x_{2} x_{5}^{2}+2 x_{3} x_{6}^{2}+(s-s \mathbf{i})\left(x_{2} x_{5}+x_{3} x_{6}\right)-s c_{3}^{0}-c_{6}^{0} \mathbf{i}-c_{5}^{0}
\end{array}\right)=0 .
$$

whose mixed volume is 8 .

For $\sigma=(1,1,1)$,

$$
Q_{\sigma}^{s}(x)=\left(\begin{array}{c}
x_{1}+x_{2}+x_{3}-c_{1}^{0} \\
x_{1}^{2}+x_{2}^{2}+x_{3}^{2}-c_{2}^{0} \\
x_{1} x_{4}+x_{2} x_{5}+x_{3} x_{6}-c_{3}^{0} \\
x_{1} x_{7}+x_{2} x_{8}+x_{3} x_{9}-c_{4}^{0} \\
x_{1} x_{4}^{2}+x_{2} x_{5}^{2}+x_{3} x_{6}^{2}-c_{5}^{0} \\
x_{1} x_{4} x_{7}+x_{2} x_{5} x_{8}+x_{3} x_{6} x_{9}-c_{6}^{0} \\
x_{4}+x_{7} \mathbf{i}+s \\
x_{5}+x_{8} \mathbf{i}+s \\
x_{6}+x_{9} \mathbf{i}+s
\end{array}\right)=0 .
$$

Eliminating $x_{7}, x_{8}, x_{9}$, and by some simple symbolic computations, (4.3) is equivalently transformed to

$$
\widehat{Q}_{\sigma}^{s}(\widehat{x})=\left(\begin{array}{c}
x_{1}+x_{2}+x_{3}-c_{1}^{0} \\
x_{1}^{2}+x_{2}^{2}+x_{3}^{2}-c_{2}^{0} \\
x_{1} x_{4}+x_{2} x_{5}+x_{3} x_{6}-c_{3}^{0} \\
c_{3}^{0} \mathbf{i}+s c_{1}^{0} \mathbf{i}-c_{4}^{0} \\
x_{1} x_{4}^{2}+x_{2} x_{5}^{2}+x_{3} x_{6}^{2}-c_{5}^{0} \\
c_{5}^{0} \mathbf{i}+s c_{3}^{0} \mathbf{i}-c_{6}^{0}
\end{array}\right)=0
$$

For the generic chosen complex numbers $c_{1}^{0}, c_{3}^{0}, c_{4}^{0}, c_{5}^{0}, c_{6}^{0}, s$,

$$
c_{3}^{0} \mathbf{i}+s c_{1}^{0} \mathbf{i}-c_{4}^{0} \neq 0, c_{5}^{0} \mathbf{i}+s c_{3}^{0} \mathbf{i}-c_{6}^{0} \neq 0 .
$$

Thus the subsystem is an "inconsistent system" and need not be solved.

\section{NumeriCAl EXPERIMENTS AND APPLICATIONS}

In this section, the symmetric hybrid method, the hybrid method and the regeneration method are respectively applied to solve several deficient polynomial systems coming from MTPS's which appeared in the literature [4,23] and the practical problem in (1.7) for $n=3,4,5,6,7$. In [31, for polynomial systems transformed from MTPS's, we have shown the hybrid method is more efficient than the polyhedral homotopy methods or the random product homotopy methods. Therefore, in this paper, we only compared our algorithm with the hybrid method and the regeneration method.

Our numerical results are obtained by PHCpack 24] running on a machine with Windows 7.0 operation system, Intel(R) Core(TM)2 Duo P8600 2.40GHz processor and $2 \mathrm{~GB}$ of memory.

Throughout the remainder of this section, it will be convenient to use the following notations. 
(1) "BN", "MBN" and "MV" respectively denote the Bézout number, the multi-homogeneous Bézout number and the mixed volume of the target system, calculated by the software PHCpack.

(2) "SN" denotes the total solution number of the target systems, and "RSN" denotes the representative solution number of the target system.

(3) "Hybrid-PH" is the elapsed time by the hybrid method, in which we apply the polyhedral homotopy method to solve the start system. and "HybridSPH" is the elapsed time by symmetric hybrid method, in which we apply the polyhedral homotopy method to solve the start system. "Bertini V1.3" is the elapsed time by the software Bertini [1, which implements the regeneration method to solve deficient polynomial systems. "HOM4PS 2.0" is the elapsed time by the software HOM4PS 2.0.

(4) " $\mathrm{N}_{\mathrm{T}}$ " is the total number of subsystems, and " $\mathrm{N}_{\mathrm{C}}$ " is the number of consistent subsystems.

Example 5.1 (d1 system in [4,23]). Consider the following polynomial system

$$
\left(\begin{array}{c}
3 x_{2}+2 x_{3}+x_{4}-3.9701 \\
3 x_{1} x_{8}+2 x_{1} x_{9}+x_{1} x_{10}-1.7172 \\
3 x_{7} x_{8}+2 x_{7} x_{9}+x_{7} x_{10}-4.0616 \\
x_{2} x_{5}+x_{3} x_{5}+x_{4} x_{5}-1.9791 \\
x_{7} x_{8} x_{5}+x_{7} x_{9} x_{5}+x_{7} x_{10} x_{5}+x_{1} x_{11}-1.9115 \\
-x_{2} x_{11} x_{6}-x_{3} x_{11} x_{6}-x_{4} x_{11} x_{6}+x_{8} x_{12}+x_{9} x_{12}+x_{10} x_{12}-0.4077 \\
x_{1}^{2}+x_{7}^{2}-1 \\
x_{2}^{2}+x_{8}^{2}-1 \\
x_{3}^{2}+x_{9}^{2}-1 \\
x_{4}^{2}+x_{10}^{2}-1 \\
x_{5}^{2}+x_{11}^{2}-1 \\
x_{6}^{2}+x_{12}^{2}-1
\end{array}\right)=0 .
$$

For the target polynomial system, we construct the start system $Q(x)=0$ as (2.7). $Q(x)=0$ can be decomposed into $2^{6}$ 6-dimensional polynomial subsystems $Q_{\sigma}(x)=0$. For $\sigma_{2}=\sigma_{3}=\sigma_{4}=1$,

$$
Q_{\sigma}(x)=\left(\begin{array}{c}
3 x_{2}+2 x_{3}+x_{4}-c_{1}^{0} \\
3 x_{1} x_{8}+2 x_{1} x_{9}+x_{1} x_{10}-c_{2}^{0} \\
3 x_{7} x_{8}+2 x_{7} x_{9}+x_{7} x_{10}-c_{3}^{0} \\
x_{2} x_{5}+x_{3} x_{5}+x_{4} x_{5}-c_{4}^{0} \\
x_{7} x_{8} x_{5}+x_{7} x_{9} x_{5}+x_{7} x_{10} x_{5}+x_{1} x_{11}-c_{5}^{0} \\
x_{1} \mathbf{i}^{\sigma_{1}+3}+x_{71} \mathbf{i}^{3 \sigma_{1}+2}+s \\
x_{8}+x_{2} \mathbf{i}+s \\
x_{9}+x_{3} \mathbf{i}+s \\
x_{10}+x_{4} \mathbf{i}+s \\
x_{5} \mathbf{i}^{\sigma_{5}+3}+x_{11} \mathbf{i}^{3 \sigma_{5}+2}+s \\
x_{6} \mathbf{i}^{\sigma_{6}+3}+x_{12} \mathbf{i}^{3 \sigma_{6}+2}+s
\end{array}\right.
$$

where $c_{1}^{0}, c_{2}^{0}, c_{3}^{0}, c_{4}^{0}, c_{5}^{0}, c_{6}^{0}, s$ are random nonzero complex numbers and $\sigma_{i} \in\{1,2\}$.

The eighth to tenth equations of $Q_{\sigma}(x)=0$ are equivalent to

$$
x_{k+6}=-x_{k} \mathbf{i}-s,
$$


for $k=2,3,4$, and by variable substitution (5.1), the second equation and the third equation in $Q_{\sigma}(x)=0$ are transformed into

$$
\left\{\begin{array}{l}
-x_{1}\left(3 x_{2}+2 x_{3}+x_{4}\right) \mathbf{i}-6 s x_{1}=c_{2}^{0}, \\
-x_{7}\left(3 x_{2}+2 x_{3}+x_{4}\right) \mathbf{i}-6 s x_{7}=c_{3}^{0} .
\end{array}\right.
$$

Because $3 x_{2}+2 x_{3}+x_{4}=c_{1}^{0}, x_{1}, x_{7}$ should satisfy

$$
\left\{\begin{array}{l}
-c_{1}^{0} x_{1} \mathbf{i}-6 s x_{1}-c_{2}^{0}=0, \\
-c_{1}^{0} x_{7} \mathbf{i}-6 s x_{7}-c_{3}^{0}=0, \\
x_{1} \mathbf{i}^{\sigma_{1}+3}+x_{7} \mathbf{i}^{3 \sigma_{1}+2}+s=0,
\end{array}\right.
$$

and the system (5.2) is inconsistent due to the randomization of $c_{1}^{0}, c_{2}^{0}, c_{3}^{0}, s$. Through use of simple analysis, we find that $Q_{\sigma}(x)=0$ has no solutions for all $\sigma \in\left\{\left(\sigma_{1}, \ldots, \sigma_{6}\right) \mid \sigma_{2}=\sigma_{3}=\sigma_{4}\right\}$. Thus the actual number of the subsystems that need to be solved is $2^{6}-2 \cdot 2^{3}=48$, and hence the number of subsystems that have to be solved is $\frac{3}{4}$ times the number of the total subsystems decomposed from the start system.

The results are given in Table 5.1a and Table 5.1b.

TABle 5.1a. Sizes of Example 5.1.

\begin{tabular}{c|c|c|c|c|c|c|c|c}
\hline $\mathrm{n}$ & $\mathrm{m}$ & $\mathrm{BN}$ & $\mathrm{MBN}$ & $\mathrm{MV}$ & $\mathrm{SN}$ & $\mathrm{RSN}$ & $\mathrm{N}_{\mathrm{T}}$ & $\mathrm{N}_{\mathrm{C}}$ \\
\hline 0 & 6 & 4608 & 320 & 192 & 48 & 48 & 64 & 48 \\
\hline
\end{tabular}

TABLE 5.1b. Elapsed time for Example 5.1.

\begin{tabular}{c|c|c|c}
\hline Hybrid-PH & Hybrid-SPH & HOM4PS 2.0 & Bertini V1.3 \\
\hline 1.732 & 1.553 & 1.765 & 10.072 \\
\hline
\end{tabular}

Example 5.2 (Polynomial system in (1.7) for $n=3, \ldots, 7$ ).

TABLE 5.2a. Sizes of Example 5.2.

\begin{tabular}{c|c|c|c|c|c|c|c|c}
\hline $\mathrm{n}$ & $\mathrm{m}$ & $\mathrm{BN}$ & $\mathrm{MBN}$ & $\mathrm{MV}$ & $\mathrm{SN}$ & $\mathrm{RSN}$ & $\mathrm{N}_{\mathrm{T}}$ & $\mathrm{N}_{\mathrm{C}}$ \\
\hline 3 & 3 & 576 & 192 & 96 & 24 & 4 & 8 & 2 \\
\hline 4 & 4 & 10368 & 2464 & 992 & 192 & 8 & 16 & 1 \\
\hline 5 & 5 & 331776 & 73728 & 14592 & 960 & 8 & 32 & 2 \\
\hline 6 & 6 & 16588800 & 3736576 & 301568 & 5760 & 8 & 64 & 1 \\
\hline 7 & 7 & 1194393600 & 270811136 & 6705152 & 40320 & 8 & 128 & 2 \\
\hline
\end{tabular}

Remark 5.1. In Example 5.2, as shown in Example 4.1, the subsystems can be considerably reduced in mixed volume, on the other hand, some subsystems are inconsistent systems and need not be solved indeed at all, and moreover, due to the symmetry, only one or two consistent systems need to be solved, the computational work is reduced greatly.

Remark 5.2. HOM4PS [7] is very efficient in applying the polyhedral homotopy method to solve polynomial systems, however, it provides less interface and is not suitable to be applied to implement our symmetric hybrid homotopy method. For the software Bertini, its Linux version is much faster than the Windows version, further comparison in the Linux system should be investigated. 
TABLE 5.2b. Elapsed time for Example 5.2.

\begin{tabular}{c|c|c|c}
\hline Hybrid-PH & Hybrid-SPH & HOM4PS 2.0 & Bertini V1.3 \\
\hline 1.545 & 0.908 & 0.578 & 10.072 \\
\hline 29.782 & 6.433 & 10.14 & 275.133 \\
\hline 460.783 & 56.342 & 250.467 & 22175.532 \\
\hline 11231.4 & 450.378 & $>10 \mathrm{~h}$ & $>20 \mathrm{~h}$ \\
\hline$>20 \mathrm{~h}$ & 22347.478 & $>20 \mathrm{~h}$ & $>20 \mathrm{~h}$ \\
\hline
\end{tabular}

\section{Conclusion}

Mixed trigonometric polynomial systems rather frequently occur in many applications, such as kinematics, mechanical system design and signal processing. A mixed trigonometric polynomial system is a polynomial system whose monomial is a mixture of some variables and sine or cosine functions applied to other variables. Through a simple variable substitution and adding $m$ quadratic equations of the form $x^{2}+y^{2}-1=0$, a mixed trigonometric polynomial system can be naturally transformed to a polynomial system. The appended $m$ quadratic equations have an inherent symmetric structure, which is difficult to be directly exploited by the existing methods for solving polynomial systems.

In this paper, to keep the symmetry of the $m$ appended equations and further the (partial) symmetry of the target system, a symmetric hybrid homotopy and hybrid polynomial system solving method are presented. The main work of our new algorithm is focused on solving the start system, due to the special product structure of the last $m$ polynomials, the work is equivalent to finding all isolated solutions of some subsystems of lower dimension, and each subsystem has the same structure, therefore, once we get all isolated solutions of a subsystem $Q_{\sigma}(x)=0$, then the coefficient-parameter homotopy or cheater's homotopy with the start system $Q_{\sigma}(x)=0$ can be applied to solve the other subsystems, and only the solution number of homotopy paths should be followed. On the other hand, by some simple symbolic computation techniques, some subsystems can be reduced in Bézout number or mixed volume, and in many cases, we can even assert that some of the reduced subsystems are inconsistent and need not to be solved indeed, and furthermore, due to the symmetry, only partial subsystems need to be solved, and in the process of path following, only the representative solution paths should be followed. Thus the computational work can be reduced greatly. For polynomial systems transformed from MTPS's, numerical results show our new algorithm performs really well and is more efficient than the hybrid method, and also the random product homotopy method, the polyhedral homotopy method and the regeneration method.

\section{REFERENCES}

[1] Daniel J. Bates, Jonathan D. Hauenstein, Andrew J. Sommese, and Charles W. Wampler, Bertini: Software for numerical algebraic geometry, http://www.nd.edu/ sommese/bertini/.

[2] David Cox, John Little, and Donal O'Shea, Using algebraic geometry, Graduate Texts in Mathematics, vol. 185, Springer-Verlag, New York, 1998. MR.1639811 (99h:13033)

[3] Robin Hartshorne, Algebraic geometry, Springer-Verlag, New York, 1977. Graduate Texts in Mathematics, No. 52. MR0463157(57 \#3116) 
[4] H. Hong and V. Stahl, Safe starting regions by fixed points and tightening, Computing 53 (1994), no. 3-4, 323-335, DOI 10.1007/BF02307383 (English, with English and German summaries). International Symposium on Scientific Computing, Computer Arithmetic and Validated Numerics (Vienna, 1993). MR1308770(95i:65081)

[5] Birkett Huber and Bernd Sturmfels, A polyhedral method for solving sparse polynomial systems, Math. Comp. 64 (1995), no. 212, 1541-1555, DOI 10.2307/2153370. MR 1297471 (95m:65100)

[6] Jonathan D. Hauenstein, Andrew J. Sommese, and Charles W. Wampler, Regeneration homotopies for solving systems of polynomials, Math. Comp. 80 (2011), no. 273, 345-377, DOI 10.1090/S0025-5718-2010-02399-3. MR2728983 (2012d:65105)

[7] T. L. Lee, T. Y. Li, and C. H. Tsai, HOM4PS-2.0: a software package for solving polynomial systems by the polyhedral homotopy continuation method, Computing 83 (2008), no. 2-3, 109-133, DOI 10.1007/s00607-008-0015-6. MR2457354(2009h:65223)

[8] T. Y. Li, Numerical solution of polynomial systems by homotopy continuation methods, Handbook of numerical analysis, Vol. XI, Handb. Numer. Anal., XI, North-Holland, Amsterdam, 2003, pp. 209-304. MR2009773 (2004k:65089)

[9] T. Y. Li, Tim Sauer, and J. A. Yorke, The cheater's homotopy: an efficient procedure for solving systems of polynomial equations, SIAM J. Numer. Anal. 26 (1989), no. 5, 1241-1251, DOI 10.1137/0726069. MR1014884(90m:65105)

[10] T.-Y. Li, Tim Sauer, and James A. Yorke, The random product homotopy and deficient polynomial systems, Numer. Math. 51 (1987), no. 5, 481-500, DOI 10.1007/BF01400351. MR.910860(88j:65108)

[11] T. Y. Li and Xiao Shen Wang, Solving deficient polynomial systems with homotopies which keep the subschemes at infinity invariant, Math. Comp. 56 (1991), no. 194, 693-710, DOI 10.2307/2008402. MR1066835 (91j:65089)

[12] Tien-Yien Li, On Chow, Mallet-Paret and Yorke homotopy for solving system of polynomials, Bull. Inst. Math. Acad. Sinica 11 (1983), no. 3, 433-437. MR.726989 (85k:58015)

[13] Tien-Yien Li, Solving polynomial systems, Math. Intelligencer 9 (1987), no. 3, 33-39, DOI 10.1007/BF03023953. MR895771 (89d:12001)

[14] Tien-Yien Li, Solving polynomial systems by polyhedral homotopies, Taiwanese J. Math. 3 (1999), no. 3, 251-279. MR1705990 (2000e:65057)

[15] Tien-Yien Li and Tim Sauer, A simple homotopy for solving deficient polynomial systems, Japan J. Appl. Math. 6 (1989), no. 3, 409-419, DOI 10.1007/BF03167887. MR.1019683 (90j:65077)

[16] Tien-Yien Li, Tim Sauer, and James A. Yorke, Numerical solution of a class of deficient polynomial systems, SIAM J. Numer. Anal. 24 (1987), no. 2, 435-451, DOI 10.1137/0724032. MR881375 (89e:90181)

[17] Pavol Meravý, Symmetric homotopies for solving systems of polynomial equations, Math. Slovaca 39 (1989), no. 3, 277-288 (English, with Russian summary). MR 1016345(91d:65074)

[18] Alexander Morgan and Andrew Sommese, Computing all solutions to polynomial systems using homotopy continuation, Appl. Math. Comput. 24 (1987), no. 2, 115-138, DOI 10.1016/0096-3003(87)90064-6. MR914807(89b:65126)

[19] Alexander Morgan and Andrew Sommese, A homotopy for solving general polynomial systems that respects $m$-homogeneous structures, Appl. Math. Comput. 24 (1987), no. 2, 101-113, DOI 10.1016/0096-3003(87)90063-4. MR914806 (88j:65110)

[20] Alexander P. Morgan, A homotopy for solving polynomial systems, Appl. Math. Comput. 18 (1986), no. 1, 87-92, DOI 10.1016/0096-3003(86)90030-5. MR815774 (87c:90194)

[21] Alexander P. Morgan and Andrew J. Sommese, Coefficient-parameter polynomial continuation, Appl. Math. Comput. 29 (1989), no. 2, 123-160, DOI 10.1016/0096-3003(89)90099-4. MR977815 (90f:58018)

[22] Alexander P. Morgan and Andrew J. Sommese, Coefficient-parameter polynomial continuation, Appl. Math. Comput. 29 (1989), no. 2, 123-160, DOI 10.1016/0096-3003(89)90099-4. MR.977815(90f:58018)

[23] Pascal Van Hentenryck, David McAllester, and Deepak Kapur, Solving polynomial systems using a branch and prune approach, SIAM J. Numer. Anal. 34 (1997), no. 2, 797-827, DOI 10.1137/S0036142995281504. MR1442939 (98g:65050)

[24] Jan Verschelde, Algorithm 795: Phcpack: A general-purpose solver for polynomial systems by homotopy continuation, 25 (1999), no. 2, 251-276. 
[25] Jan Verschelde, Marc Beckers, and Ann Haegemans, A new start system for solving deficient polynomial systems using continuation, Appl. Math. Comput. 44 (1991), no. 3, 225-239, DOI 10.1016/0096-3003(91)90059-V. MR 1115067 (92c:65068)

[26] Jan Verschelde and Ronald Cools, Symmetric homotopy construction, Proceedings of the Fifth International Congress on Computational and Applied Mathematics (Leuven, 1992), 1994, pp. 575-592, DOI 10.1016/0377-0427(94)90329-8. MR.1284290 (95j:65056)

[27] Jan Verschelde and Karin Gatermann, Symmetric Newton polytopes for solving sparse polynomial systems, Adv. in Appl. Math. 16 (1995), no. 1, 95-127, DOI 10.1006/aama.1995.1005. MR1317613 (95m:65101)

[28] Jan Verschelde and Ann Haegemans, The GBQ-algorithm for constructing start systems of homotopies for polynomial systems, SIAM J. Numer. Anal. 30 (1993), no. 2, 583-594, DOI 10.1137/0730028. MR 1211406 (94b:65075)

[29] Jan Verschelde, Pierre Verlinden, and Ronald Cools, Homotopies exploiting Newton polytopes for solving sparse polynomial systems, SIAM J. Numer. Anal. 31 (1994), no. 3, 915-930, DOI 10.1137/0731049. MR 1275120 (94m:65084)

[30] Alden H. Wright, Finding all solutions to a system of polynomial equations, Math. Comp. 44 (1985), no. 169, 125-133, DOI 10.2307/2007797. MR771035 (86i:12001)

[31] Bo Yu and Bo Dong, A hybrid polynomial system solving method for mixed trigonometric polynomial systems, SIAM J. Numer. Anal. 46 (2008), no. 3, 1503-1518, DOI 10.1137/070681740. MR2391004 (2009b:65133)

[32] Bo Yu and Guo Chen Feng, The random product homotopy for solving polynomial systems in $\mathbf{C}^{k_{1}} \times \mathbf{C}^{k_{2}} \times \cdots \times \mathbf{C}^{k_{m}}$, Computer mathematics (Tianjin, 1991), Nankai Ser. Pure Appl. Math. Theoret. Phys., vol. 5, World Sci. Publ., River Edge, NJ, 1993, pp. 36-45. MR 1257442 (95c:65080)

[33] Walter Zulehner, A simple homotopy method for determining all isolated solutions to polynomial systems, Math. Comp. 50 (1988), no. 181, 167-177, DOI 10.2307/2007920. MR917824 (89b:65130)

School of Mathematical Sciences, Dalian University of Technology, Dalian, LiaonING 116024, PeOple's RePublic of ChinA

E-mail address: dongbo@dlut.edu.cn

School of Mathematical Sciences, Dalian University of Technology, Dalian, LiaonING 116024, People's Republic of China

E-mail address: yubo@dlut.edu.cn

Faculty of Sciences, Shenyang Agricultural University, Shenyang, Liaoning, 110866, People's Republic of China

E-mail address: yuyan1015@syau.edu.cn 